\title{
A Telemedicine-Guided Self-Collection Approach for PCR-Based SARS-CoV-2 Testing: Comparative Study
}

Silvia Würstle ${ }^{1^{*}}, \mathrm{MD}$, Dr med; Johanna Erber ${ }^{1^{*}}, \mathrm{MD}$, Dr med; Michael Hanselmann ${ }^{2}, \mathrm{MSc}, \mathrm{PhD}$; Dieter Hoffmann ${ }^{3}$, MD, Dr med; Stanislas Werfel ${ }^{4}$, MD, Dr med; Svenja Hering ${ }^{3}$, MA; Simon Weidlich ${ }^{1}$, MD, Dr med; Jochen Schneider ${ }^{1}$, MD, Dr med; Ralf Franke ${ }^{5}$, Dr med; Michael Maier ${ }^{5}$; Andreas G Henkel ${ }^{6}$; Roland M Schmid ${ }^{1}$, Prof Dr med; Ulrike Protzer $^{3,7}$, Prof Dr med; Michael Laxy ${ }^{2 *}$, Prof Dr; Christoph D Spinner ${ }^{1,7^{*}}$, MD, Dr med

\footnotetext{
${ }^{1}$ Department of Internal Medicine II, School of Medicine, University Hospital rechts der Isar, Technical University of Munich, Munich, Germany

${ }^{2}$ Department for Sport and Health Sciences, Professorship of Public Health and Prevention, Technical University of Munich, Munich, Germany

${ }^{3}$ School of Medicine / Helmholtz Zentrum München, Institute of Virology, Technical University of Munich, Munich, Germany

${ }^{4}$ Department of Nephrology, School of Medicine, University Hospital rechts der Isar, Technical University of Munich, Munich, Germany

${ }^{5}$ Siemens AG, Munich, Germany

${ }^{6}$ Department of Information Technology, School of Medicine, University Hospital rechts der Isar, Technical University of Munich, Munich, Germany

${ }^{7}$ German Centre for Infection Research (DZIF), Partner site, Munich, Germany

* these authors contributed equally
}

\section{Corresponding Author:}

Christoph D Spinner, MD, Dr med

Department of Internal Medicine II, School of Medicine

University Hospital rechts der Isar

Technical University of Munich

Ismaninger Str 22

Munich, 81675

Germany

Phone: 498941404375

Email: Christoph.Spinner@mri.tum.de

\section{Abstract}

Background: Large-scale, polymerase chain reaction (PCR)-based SARS-CoV-2 testing is expensive, resource intensive, and time consuming. A self-collection approach is a probable alternative; however, its feasibility, cost, and ability to prevent infections need to be evaluated.

Objective: This study aims to compare an innovative self-collection approach with a regular SARS-CoV-2 testing strategy in a large European industrial manufacturing site.

Methods: The feasibility of a telemedicine-guided PCR-based self-collection approach was assessed for 150 employees (intervention group) and compared with a regular SARS-CoV-2 testing approach used for 143 employees (control group). Acceptance, ergonomics, and efficacy were evaluated using a software application. A simulation model was implemented to evaluate the effectiveness. An interactive R shiny app was created to enable customized simulations.

Results: The test results were successfully communicated to and interpreted without uncertainty by $76 \%(114 / 150)$ and $76.9 \%$ $(110 / 143)$ of the participants in the intervention and control groups, respectively $(P=.96)$. The ratings for acceptability, ergonomics, and efficacy among intervention group participants were noninferior when compared to those among control group participants (acceptability: $71.6 \%$ vs $37.6 \%$; ergonomics: $88.1 \%$ vs $74.5 \%$; efficacy: $86.4 \%$ vs $77.5 \%$ ). The self-collection approach was found to be less time consuming ( $23 \mathrm{~min}$ vs $38 \mathrm{~min} ; P<.001$ ). The simulation model indicated that both testing approaches reduce the risk of infection, and the self-collection approach tends to be slightly less effective owing to its lower sensitivity.

Conclusions: The self-collection approach for SARS-CoV-2 diagnosis was found to be technically feasible and well rated in terms of acceptance, ergonomics, and efficacy. The simulation model facilitates the evaluation of test effectiveness; nonetheless, considering context specificity, appropriate adaptation by companies is required. 


\section{KEYWORDS}

self-sampling; telemedicine; test strategy effectiveness; simulation model; SARS-CoV-2; COVID-19

\section{Introduction}

Numerous campaigns for COVID-19 vaccination have been initiated worldwide, but the pandemic continues to spread. Emerging variants of SARS-CoV-2, as well as reports of breakthrough infections, underline that public health mitigation measures, including testing strategies, need to be continued. In terms of sensitivity, nucleic acid amplification tests (NAATs) are standard for SARS-CoV-2 detection in respiratory samples obtained by medical personnel (ie, regular testing approach) [1]. However, the implementation of professional, large-scale routine testing is limited owing to high organizational costs and intensive efforts entailed. The authorization of lateral-flow SARS-CoV-2 antigen tests enables the implementation of self-testing strategies (ie, self-testing approach), which improves the turnaround times of test results. Due to the absence of amplification steps, the analytic sensitivity of lateral-flow antigen tests is substantially inferior to that of a NAAT-based approach, particularly when the viral load is low in early or late stages of disease progression [2].

A self-collection approach based on NAAT performed on self-collected swabs could combine the advantages of regular testing and self-testing approaches. Previous studies have shown that the sensitivity of SARS-CoV-2 polymerase chain reaction (PCR) testing using self-collected swabs was comparable to swabs collected by health care professionals, with an acceptable impact on the test sensitivity [3-7]. Self-collection reduces the use of resource-intensive testing centers and personal protective equipment, and it eliminates the requirement of swab collection by medical personnel. Furthermore, the willingness of individuals to undergo testing might be increased as the time required for testing is expected to be lesser, and self-collected swabs are considered to be more convenient for the operator [8]. Structured evaluations of different testing strategies are required to compare their feasibility, costs, and infection-prevention capabilities.

In this prospective, two-arm feasibility study, we aimed to compare a telemedicine-guided self-collection approach with a regular testing approach involving a sample collected by a health care professional for PCR-based SARS-CoV-2 diagnostics, primarily focusing on the feasibility, and secondarily on the acceptance, ergonomics, and efficacy of the testing strategy implemented onsite at a large European industrial manufacturing company in Germany. For the self-collection approach, we developed a telemedicine-guided approach, which included obtaining electronic consent, electronic registration, and communication of the SARS-CoV-2 PCR test result. Testing approaches in companies aim to reduce the infection risk arising from undetected but infectious employees. In this study, none of the SARS-CoV-2 PCR tests performed in the employees returned positive. We performed health economic modeling to analyze potential effects of different testing strategies and developed a shiny app to enable people to run simulations using different medical assumptions.

\section{Methods}

\section{Study Design and Participants}

The process flow of this prospective, interventional, open-label, controlled, two-arm feasibility trial is illustrated below (Figure 1). Between November 11, 2020, and December 11, 2020, all employees of Siemens F80, SYKATEC GmbH, and Valeo Siemens eAutomotive Germany $\mathrm{GmbH}$ were invited to participate in this study (see Figure $\mathrm{S} 1$ in Multimedia appendix 1 for the advertisement flyer). The main inclusion criteria were the ability to download and use the user application of the software app principa (PlanOrg $\mathrm{GmbH}$ ), which is part of the hospital information system and clinical workplace system at the University Hospital rechts der Isar (Technical University of Munich, Germany). The app could be downloaded using a study-specific quick response $(\mathrm{QR})$ code with a deep link to the Android or Apple store. Upon registration, electronic consent was obtained from all participants, following which they were randomized 1:1 into two study groups using the Java function SecureRandom (algorithm: SHA1PRNG). Participants in group 1 (intervention group; telemedicine-guided SARS-CoV-2 diagnostic testing with self-collection) were asked to collect a prepacked self-collection kit, including the United Nations' recommendation of dangerous goods (UN 3373) compliant packaging kit (cardboard and container) with a prepaid shipping label, printed instructions (see Figure S2 in Multimedia Appendix 1), and a swab (FLOQSwabs 552C Regular Flocked Swab with an 80-mm breakpoint, Copan). The risk factors for and the symptoms of SARS-CoV-2 infection were self-reported using the app (see Table S1 in Multimedia Appendix 1). A short explanatory video was made available in the app for demonstration to the participants (see Multimedia Appendix 2). Participants self-collected an oropharyngeal swab, scanned the individualized unique code of the shipping kit, and mailed the kit to the study center at University Hospital rechts der Isar for analysis. SARS-CoV-2 PCR test results were provided via the app; in addition, the PDF report could be exported for personal use. Group 2 participants (control group; regular testing approach via appointment at the test center) were asked to book an appointment via phone for SARS-CoV-2 testing at a test center located at the study site. After assessment of SARS-CoV-2-specific risks and symptoms by a staff member, a nasopharyngeal sample was collected (REST Clinical Virus Transport Medium [CTM] swab, Rapid \& Easy System Technology; Noble Biosciences, Inc.) by trained medical staff. The samples were shipped to the study center and processed as described for group 1. SARS-CoV-2 PCR test results were exclusively provided by phone and without any hard copy. Upon communication of the test results, participants in both groups were asked to evaluate the respective testing strategy and app by using a visual slider on a 7 -point Likert scale $(0=$ strong disagreement, $6=$ strong agreement) within the app. The questionnaire items were based on the National Aeronautics and Space Administration (NASA) Task Load Index [9] for evaluating the effort and the Website Analysis and Measurement 
Inventory for evaluating user satisfaction. The time required minutes. The completion of the questionnaire was facultative. for the entire sample collection procedure was assessed in

Figure 1. Process flow. Upon registration and informed consent, participants were randomized into a self-collection (intervention) and regular testing (control) group. Swabs from participants of both groups were submitted to the study center and SARS-CoV-2 polymerase chain reaction (PCR) was performed. Questionnaire on the symptoms and risk factors was completed before conducting the procedure, whereas the questionnaire on user satisfaction was completed after the study procedure. The term 'app' refers to the software application principa (PlanOrg GmbH, Jena, Germany).

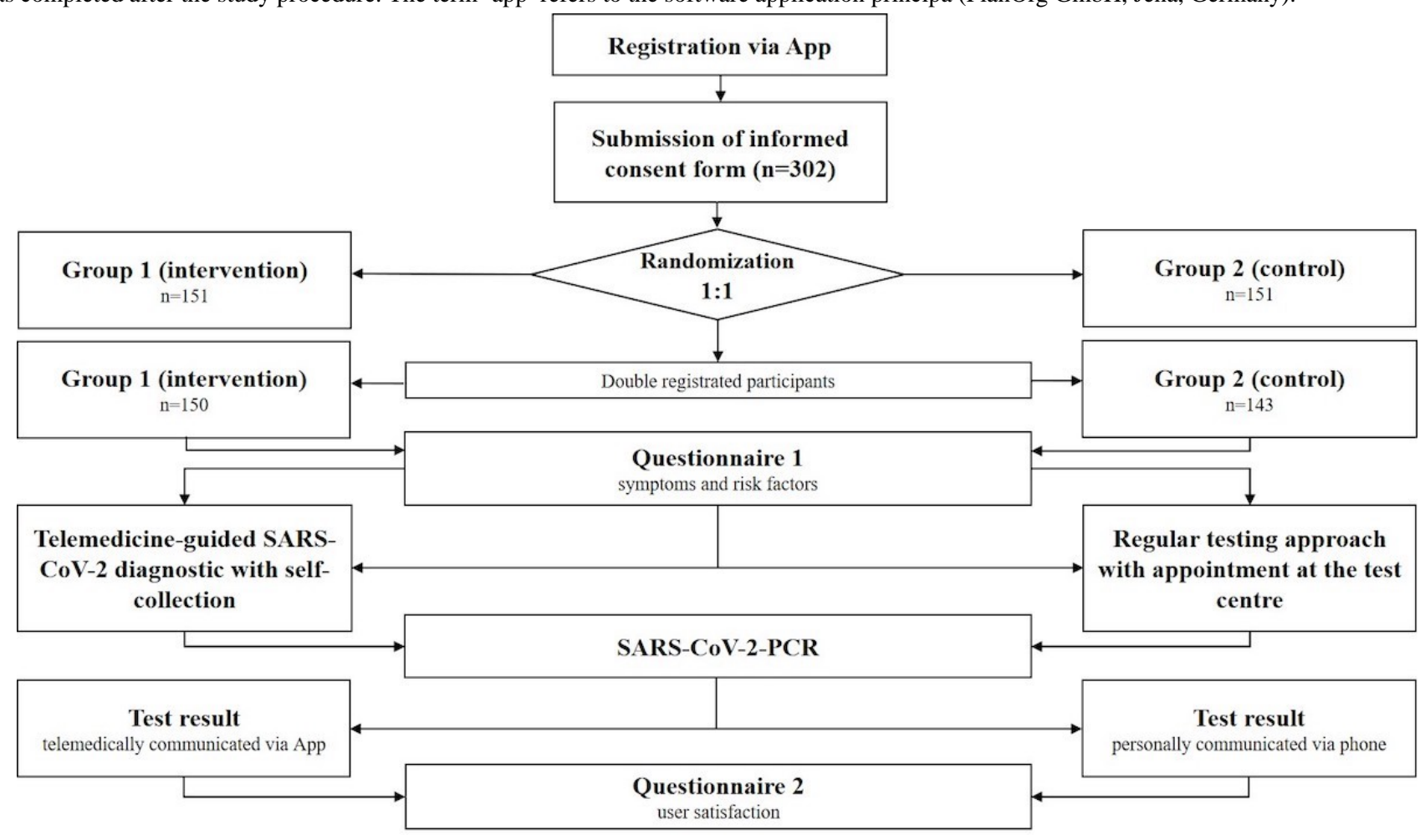

\section{Ethics Approval}

This study was approved by the ethics committee at the Technical University of Munich, School of Medicine, University Hospital rechts der Isar, Munich, Germany (approval 603/20-SH) and conducted in accordance with the Declaration of Helsinki.

\section{Primary and Secondary End Points}

The primary end point was the proportion of participants for whom self-collection, virological diagnosis, and reporting of the test result were successfully conducted, and the statements made by the participants in response to the questionnaire did not indicate any uncertainty with respect to interpretation of the test results (see Table $\mathrm{S} 2$ in Multimedia Appendix 1 for details). The secondary end points were (1) the proportion of participants for whom virological findings were available but uncertainty regarding the interpretation was reported, and (2) patient-reported outcomes, including acceptance, ergonomics, and efficacy. The statements of group-specific questionnaires were assigned to these three outcomes (see Table S4 in Multimedia Appendix 1). Threshold values were defined to transfer the ratings into a dichotomous scale (favorable rating $>3$ points; unfavorable rating $\leq 3$ points). A favorable rating for more than $70 \%$ of all the statements was interpreted as a satisfactory outcome.

\section{Diagnostic Procedures}

All virological diagnostics were performed by the expert staff of the Institute of Virology, Technical University of Munich. Nucleic acids were extracted using the mSample Preparation System DNA kit (Promega), and a standard protocol was followed on an m2000sp device for RNA and DNA extraction (Abbott). SARS-CoV-2_N1 and SARS-CoV-2_N3 primer and probe sets were used for amplification on an ABI 7500 real-time PCR cycler (Thermo Fisher Scientific), following the protocol of the Division of Viral Diseases, National Center of Immunization and Respiratory Diseases, Centers for Disease Control and Prevention (accreditation authority No. D-ML-14063-02-00) [10].

\section{Sample Size Calculation}

For calculation of the sample size, we assumed that $50 \%$ of the participants met the criteria for the primary end point. A confidence level of $95 \%$ and a specified CI of 0.08 resulted in 151 participants per group $(n=302)$. Dropouts were not considered because they were represented in the fraction of the participants to whom the test results were not successfully communicated. Recruitment was stopped when the calculated sample size was reached.

\section{Simulation Model for Evaluation of the Effectiveness of Test Strategy}

We developed a simulation model that facilitates evaluation of the effectiveness of distinct COVID-19 test strategies. We modeled the risk of infection that arises from individuals with 
SARS-CoV-2 infection who do not present typical COVID-19 symptoms. This implies that the model solely focuses on the risk of infection that arises from undetected, presymptomatic, and asymptomatic COVID-19 cases. Test strategies that are targeted on individuals without COVID-19 symptoms represent a measure taken to reduce this risk. The developed simulation model considers a period of 4 weeks ( 28 days) and relies on several medical assumptions. Based on expert ratings and a review of the relevant literature, we assumed that COVID-19 symptoms appear on the third day of infection [11,12]. We assumed 20\% asymptomatic infections [13] and postulated that infected individuals are infectious for 10 days [14]. Furthermore, we assumed a 7-day incidence rate of 100 and $20 \%$ immune individuals. The main outcome of the simulation model was the average number of infectious but undetected individuals working onsite per day, which can be interpreted as a measure of the risk of formation of infection clusters within the company. Considering a scenario without any testing strategy as the benchmark, the model allows us to calculate and compare the measurements of relative risk reduction for different testing strategies. In our simulation model, test strategies are defined by five parameters. For this study, we considered a population of 10,000 individuals (workers) and assumed that $80 \%$ of susceptible individuals actually participated in the testing program. In addition, we assumed that participants were tested once per week. We assumed these parameters to be equal for both testing programs used in this study. The other two parameters defining a testing program are closely related to the test performed and are therefore of particular importance in this study. The first parameter is test sensitivity, which is supposed to be higher in the case of the regular testing approach. Prior research indicates a sensitivity of $90 \%$ for the regular testing approach and $80 \%$ for the self-collection approach [5-7,15]. However, because the exact difference remains unknown, we report simulation results for four different levels of test sensitivity: $70 \%, 80 \%, 90 \%$, and $100 \%$. The second parameter that is relevant in this study is the number of days taken to communicate the test results to the program participants (ie, turnaround time). Unfortunately, the trial conducted does not provide a clear indication of the turnaround time of both testing approaches. In fact, the turnaround times may differ between the two approaches. Therefore, we ran our simulations for four different turnaround times: $0,1,2$, and 3 . Because we differentiate between the four sensitivity levels and the four turnaround times, we report the results for a total of 16 simulation scenarios. The comparison of the obtained results allows us to gain insights into the potential effectiveness of the testing approaches. Due to the stochastic nature of our simulation model, the result of one simulation run is subject to uncertainty. To address this uncertainty, we ran each of our 16 simulations 1000 times, and accordingly, the mean of the obtained simulation results is presented.

Few of our assumptions and model input parameters might vary from time to time and/or are context specific. Therefore, we developed an interactive R Shiny web application (R Studio version 4.0.5; $\mathrm{R}$ Foundation for Statistical Computing), which enables users to customize the model input parameters and run simulations on their own. The developed simulation tool is integrated in a website. The website also contains a detailed description of the simulation model and displays the $\mathrm{R}$ function written and used to run the simulations in this study.

It must be noted that the potential reduction in the risk implied by the simulation results is only applicable if the employees follow hygiene standards as if there were no testing programs implemented at their company. Furthermore, large-scale testing of asymptomatic individuals in a situation of low COVID-19 prevalence will lead to a large share of false-positive test results, which might necessitate the unjustified quarantining of many individuals. This potentially leads to a productivity loss and might disturb the employees unnecessarily. The declining rigorousness of the hygiene measures or the lack of trust in the test results could decrease the effectiveness of the testing strategies. Because the effectiveness of risk reduction measures depends on individual compliance, every large-scale testing program should be accompanied by an information campaign explaining the interpretation and the consequences of positive and negative test results. The developed simulation tool was integrated in a website [16].

\section{Statistical Methods}

The distributions of quantitative and qualitative data are presented as the absolute and relative frequencies or medians (range), respectively. Fisher's two-sided exact test or Pearson's chi-squared test were performed on the categorical variables, and Wilcoxon rank-sum test was performed on quantitative parameters. Statistical hypothesis testing was performed on the two-sided exploratory 0.05 significance levels. RStudio (version 4.0.2; R Foundation for Statistical Computing) was used for all statistical analyses.

\section{Availability of Data and Material}

All self-collection instructions, questionnaires, and comments of the questionnaires are provided in Multimedia Appendices 1 and 2. All raw data are available from the corresponding author on request.

\section{Results}

\section{Baseline Characteristics of Study Cohort}

In this study, 302 employees registered and consented for participation. In all, data of 293 unique participants were available for analysis because 9 employees registered twice, as duplicate registrations were not technically prevented by the app. Groups 1 and 2 consisted of 150 and 143 participants, respectively, of which $21.3 \% \quad(n=32)$ and $18.2 \% \quad(n=26)$, respectively, were female. The median age for both the groups was 42 years (range: 20-61 years in group 1 and 23-64 years in group 2). The completion rate of the symptom and risk factor questionnaire (see Table $\mathrm{S} 1$ in Multimedia Appendix 1) was $64 \%(\mathrm{n}=96)$ in group 1 and $45.4 \%(\mathrm{n}=65)$ in group 2 . In group $1,51 \%(n=49)$ of the participants reported at least one and $21 \%$ $(\mathrm{n}=20)$ of the participants reported at least two typical symptoms of COVID-19 (ie, fatigue, tiredness, cough, shortness of breath, rhinitis, loss of smell, loss of taste, sore throat, headache, limb pain, shivering, diarrhea, elevated temperature, and temperature $\geq 38^{\circ} \mathrm{C}$ ), in contrast to at least one reported symptom in $29 \%$ $(n=19)$ and at least two reported symptoms in $2 \%(n=1)$ of group 2 participants. This resulted in significantly fewer symptomatic 
participants in group $2(P=.01)$ during the assessment. Similarly, group 1 participants reported significantly more symptoms for the last 48 hours and 14 days $(P<.001)$. Predefined risk factors (ie, active or past history of smoking, cardiovascular disease, diabetes mellitus, immunosuppressive therapy, and immunodeficiency) for severe COVID-19 were reported by $44 \%(n=42)$ of group 1 participants and $60 \%(n=39)$ of group 2 participants $(P=.06)$.

\section{Primary Study End Point}

SARS-CoV-2 test results were successfully communicated to a total of 270 participants without any significant difference in the results between the two groups $(139 / 150,92.7 \%$ in group 1 vs $131 / 143,91.6 \%$ in group 2$)$. The results were not successfully communicated to $7.3 \%(11 / 150)$ and $8.4 \%(12 / 143)$ of the participants in groups 1 and 2 , respectively $(P=.91)$. None of the SARS-CoV-2 PCR results returned positive. The proportion of participants with positive primary study end point (ie, test results were successfully transmitted and participants' responses to the questionnaire did not indicate uncertainty with respect to the interpretation of the test result; see Table S2 in Multimedia Appendix 1) was 76\% (114/150) in group 1 and $76.9 \%(110 / 143)$ in group $2(P=.96)$. The median age of the participants with positive primary end point was 41 (range 20-61) years in group 1 and 43 (range 23-63) years in group 2.

\section{Secondary Study End Points}

A questionnaire evaluating user satisfaction (see Table S2 in Multimedia Appendix 1) was completed by $73.3 \%$ (110/150) of the participants in group 1 (response rate: $87 / 118,73.7 \%$ for male; $23 / 32,71.9 \%$ for female participants) and $71.3 \%$ $(102 / 143)$ of the participants in group 2 (response rate: $85 / 117$, $72.6 \%$ for male and $17 / 26,65.4 \%$ for female participants). Of all the participants evaluated for user satisfaction, $16.7 \%(n=25)$ in group 1 and $14.7 \%(n=21)$ in group 2 indicated uncertainty regarding the test result. The age was comparable to that of participants with a positive primary end point: 42 (range 26-60) years for group $1(P=.30)$ and 38 (range 24-64) years group 2 $(P=.24)$. Furthermore, gender was not significantly related to the reporting of uncertainty about the virological test result obtained in either group (group 1: $P=.20$; group 2: $P=.99$ ).

Based on the assessment of the responses to the questionnaire, acceptance was favorably rated by $71.6 \%$ (78/109), ergonomics by $88.1 \%(96 / 109)$, and efficacy by $86.4 \%(95 / 110)$ of the participants in group 1 (see Table S4 in Multimedia Appendix 1). Age was not significantly associated with the favorable evaluation of any of the outcomes (acceptance: $P=.41$, ergonomics: $P=.30$, efficacy: $P=.71$ ). Further, the evaluation of outcomes was not significant with respect to gender (women compared with men, acceptance: $P=1.0$, ergonomics: $P=.73$, efficacy: $P=.73$ ). In group 2 , acceptance of the regular testing approach was favorably evaluated by $37.6 \%$ (38/101), ergonomics by $74.5 \%$ (76/102), and efficacy by $77.5 \%$ (79/102) of the participants. The study procedure was estimated to consume an average of 23 (median 15, range 5-90) minutes by participants in group 1 compared with 38 (median 30, range 3 -180) minutes for those in group $2(P<.001)$.

\section{Analysis of Program Effectiveness}

A simulation model was developed to evaluate the effect of COVID-19 test strategies on the infection risk arising from undetected but infectious employees. We ran 16 simulations for four different levels of the test sensitivity and four different turnaround times. The mean relative risk reduction scores and the corresponding $95 \%$ CIs for these 16 simulations are tabulated below (Table 1). The results shown in the table can be interpreted as follows: given the assumptions described in the Methods section, a test strategy using a SARS-COV-2 PCR test with a sensitivity of $90 \%$ and featuring a turnaround time of 1 day has the potential to decrease the risk of infection posed by undetected but infectious workers onsite by $17.89 \%$. All other aspects remaining constant, a higher test sensitivity or a lower turnaround time increases the effectiveness of the testing strategy. Assuming that the turnaround time is comparable for both the regular approach and the self-collection approach, one might conclude that the regular approach is slightly more effective than the self-collection approach. Depending on the turnaround time selected, the difference in the relative risk reduction score ranges between $1.21 \%$ and $3.29 \%$. The self-collection approach would be more effective than the regular testing approach, if the turnaround time in the self-collection approach is 1 day lesser than that of the regular approach. To enable people to run simulations with different medical assumptions and to evaluate alternative testing strategies, we developed an interactive R Shiny app [16].

Table 1. Simulation results for relative risk reduction and impact of test sensitivity and turnaround time. Each cell of the table depicts the mean of 1000 relative risk reduction scores and the corresponding CI. In each simulation run, a population of 10,000 individuals was considered. The assumptions (see Methods section) include that $80 \%$ of the susceptible individuals participate in the testing program once a week. Turnaround time refers to the time taken to communicate the test result after testing.

\begin{tabular}{|c|c|c|c|c|}
\hline \multirow[t]{2}{*}{ Turnaround time (days) } & \multicolumn{4}{|c|}{ Relative risk reduction across various levels of test sensitivity $(\%)$, mean $(95 \% \mathrm{CI})$} \\
\hline & $100 \%$ & $90 \%$ & $80 \%$ & $70 \%$ \\
\hline 0 & $27.64(27.24-28.05)$ & $25.22(24.81-25.63)$ & $21.93(21.53-22.32)$ & $19.28(18.91-19.65)$ \\
\hline 1 & $20.08(19.72-20.43)$ & $17.89(17.53-18.24)$ & $15.75(15.40-16.08)$ & $14.02(13.68-14.35)$ \\
\hline 2 & $14.13(13.81-14.44)$ & $12.72(12.41-13.02)$ & $11.61(11.32-11.91)$ & $9.83(9.54-10.11)$ \\
\hline 3 & $10.51(10.25-10.77)$ & $9.68(9.43-9.93)$ & $8.47(8.23-8.71)$ & $7.27(7.06-7.49)$ \\
\hline
\end{tabular}




\section{Discussion}

This study aimed to compare the feasibility, acceptance, ergonomics, and efficacy of an innovative telemedicine-guided self-collection approach with a regular SARS-CoV-2 testing approach implemented onsite at a large European industrial manufacturing company in Germany.

\section{Principal Results}

Given the comparable rates of successful communication of test results in both the study groups, our data show that the telemedicine-guided self-collection approach for SARS-CoV-2 diagnostics, including registration, swab self-collection, shipping, and communication of SARS-CoV-2 PCR test result, is technically feasible. The ratings of acceptability, ergonomics, and efficacy for the self-collection approach were noninferior compared with those of the regular testing approach, with the limitation that the ratings were based on different questions for groups 1 and 2 (see Table S2 in Multimedia Appendix 1). Furthermore, the self-collection approach was rated as significantly less time-consuming than the regular testing approach. Neither gender nor age had an effect on the uncertainty regarding the study results or the acceptability, ergonomics, and efficiency ratings. Moreover, a favorable rating for acceptance and efficacy did not affect the outcome and performance of the study procedures, suggesting that a telemedicine-guided self-collection approach for SARS-CoV-2 diagnostics can be applied even among less motivated individuals.

Intriguingly, none of the 293 SARS-CoV-2 PCR tests returned positive. This might be ascribed to a low pretest probability, because all the employees were invited to participate, independent of any prevalent risk contacts or COVID-19 symptoms. Furthermore, the diagnostic laboratory was working at capacity during the second wave of the pandemic, and specimens from patients were prioritized, leading to a delay of study-specific diagnostics and the possible degradation of viral nucleic acid.

Our simulation results suggest that both the testing approaches have the potential to reduce the risk of infection posed by infectious but undetected individuals. Owing to lower sensitivity of self-collected swabs [3], the self-collection approach tends to be slightly less effective than the regular testing approach, whereas the time taken to communicate the test result is the same for both approaches. However, due to the high workload of the virological laboratory at the time of this study, we could not assess the time taken to communicate the test result, hindering a clear assessment of the testing approaches used in this study.

The costs pertaining to the investigated testing approaches vary depending on context-specific factors, including the costs for PCR tests, supply of medical staff, information technology (IT) infrastructure, and onsite time associated with testing. Therefore, a detailed cost evaluation is not within the scope of this study. However, to estimate the costs pertaining to the two testing approaches examined in this study, we conducted back-of-the-envelope calculations for the large central European manufacturing company. Interestingly, the costs of the two testing approaches mainly differ with respect to the five types of costs: costs for medical staff, hygiene costs, productivity loss, shipping costs, and IT costs. The proportion of these five types of costs indicates the testing approach that is more cost effective. The regular testing approach showed higher costs for medical staff, hygiene, and productivity loss than the self-collection approach. In contrast, the self-collection approach entailed higher shipping costs and costs for setting up and operating the app than the regular testing approach. The main share of the app costs was fixed, however, leading to a decrease in the costs per test in proportion to the number of tests conducted in the case of the self-collection approach. Therefore, the self-collection approach might be more favorable for large testing programs. Our back-of-the-envelope calculations indicate that the self-collection approach can be substantially less expensive than the regular approach for large testing programs. However, we recommend that the companies calculate the cost of testing approaches individually, considering context-specific factors.

\section{Limitations}

This study has several limitations. The age and gender did not significantly differ between the two study cohorts, suggesting an appropriate comparability of the results. However, the male participants outnumbered the female participants, which can be ascribed to a predominantly higher male workforce at the Siemens site. The study cohort did not include participants above 64 years of age. The questionnaires on user satisfaction were not completed by 40 and 41 participants in group 1 and group 2, respectively. In future studies, efforts should be made to increase the questionnaire completion rates. The primary end point was defined both as the successful communication of the test result and the lack of reported uncertainty regarding the test result in the questionnaire (see Table S2 in Multimedia Appendix 1). We note that the user satisfaction questionnaires were not completed by all participants. Therefore, it is possible that few participants might have felt insecure regarding their test results and avoided reporting it. To assess the validity of our results, we repeated our analyses by assuming that the nonresponse to the questionnaire is equivalent to the uncertainty regarding the test results. This approach did not significantly change the results of our primary analysis (see Table S5 in Multimedia Appendix 1). COVID-19 symptoms were less often reported in group 2, which might be attributed to nondisclosure of symptoms at the workplace. The risk factors for COVID-19 were more frequently reported in group 2, which is possibly related to assessment by the medical staff. The responses in the questionnaire for symptoms and risk factors were incompletely transmitted by the app for few participants in group 2 . Furthermore, the software app should be upgraded to prevent double registrations. Due to the abovementioned high workload in the virological laboratory at the time the study was performed, turnaround times were delayed, and the results could not be provided in 23 cases $(7.8 \%)$, which might have affected the satisfaction of those participants. Four participants commented that the alignment of the Likert scale was not clear; however, the interpretation of all the participants was correct. The ambiguity was clarified in the app during the course of the study. 
Nonetheless, we cannot exclude that the visual slider was occasionally moved inversely.

The simulations conducted to assess the effectiveness of the regarded testing approaches rely on several assumptions that are partially based on the expert ratings of the participating investigators. Therefore, bias cannot be excluded. Furthermore, the simulation model assumed few factors, such as a constant incidence rate and immunity rate within the specific period of 4 weeks. The developed simulation model as well as the developed Shiny app exclusively focuses on the risk of infection posed by undiagnosed but infectious individuals. Superspreading events as well as secondary transmissions by index patients were not considered in this model.

\section{Comparison With Prior Work}

Several recent studies evaluated the diagnostic reliability of self-collected versus professional-collected specimen for the detection of SARS-CoV-2 and found comparable sensitivities [3-7]. To the best of our knowledge, telemedicine-guided self-collection approaches in a home-based setting followed by PCR-based SARS-CoV-2 testing have not been investigated to date.

\section{Conclusions}

This study provides evidence that a telemedicine-guided self-collection approach for SARS-CoV-2 diagnostic testing is technically feasible, and this approach is favorably rated in terms of acceptance, ergonomics, and efficiency. Our data indicate that the resources, expense of time and labor, and personal contacts can be considerably reduced through a telemedicine-guided, self-collection approach when compared with a regular PCR-based testing strategy. Nonetheless, the risk reduction in a self-collection approach is expected to be slightly lower because the test sensitivity of the self-collected swabs is inferior to that of professional-collected swabs. Self-test approaches based on lateral-flow antigen tests may be a cost-effective alternative to PCR-based strategies and should be investigated in future studies because the test sensitivity appears to be secondary to the turnaround time regarding the risk reduction. The app-based platform we provide here may serve as the basis for enhanced connectivity in future digital approaches of personalized medicine. Indeed, the easy-to-use design combined with potential coupling with other health care interfaces may provide benefits beyond the COVID-19 pandemic.

\section{Acknowledgments}

This study was funded by the Technical University of Munich with a restricted grant of Siemens AG, Germany. Swabs were provided by Copan Diagnostics, Brescia, Italy, and were free of charge. We are thankful to all the study participants who consented to participate in this study and thank all technical staff of the Institute of Virology for their assistance in performing the diagnostic tests. We thank all the members of our study team, particularly Stefanie Gladis, Jennifer Lieb, and Christina Wernle for their assistance in conducting this study. We acknowledge all the involved members of the IT department and the software development teams for the PlanOrg and Siegele Software for their assistance.

\section{Authors' Contributions}

CDS, UP, ML, and S Würstle conceived and designed the study. S Werfel produced the instruction video. S Würstle, RF, MM, and DH were involved in the practical part of the study. DH and UP performed laboratory analysis and interpretation. S Würstle, $\mathrm{SH}$, and DH acquired the data; CDS verified the collected data together with S Weidlich and AH. S Würstle, SH, and JH analyzed and interpreted the data. MH and ML contributed to the health economic aspects. MH developed the R Shiny app. JE, MH, S Würstle, and CDS were the major contributors in writing the manuscript. ML, UP, CDS, JS, S Weidlich, S Werfel, AH, and RMS revised the manuscript. All authors read and approved the final manuscript.

\section{Conflicts of Interest}

CDS reports grants, personal fees, and nonfinancial support from AbbVie, Apeiron, Gilead Sciences, Janssen-Cilag, GSK/ViiV Healthcare, and MSD; grants and personal fees from BBraun and Eli Lilly; grants from Cepheid; and personal fees from Formycon-outside the submitted work. S Weidlich reports personal fees and nonfinancial support from Gilead Sciences and Janssen-Cilag outside the submitted work. All other authors declare that they have no conflicts of interest.

\section{Multimedia Appendix 1}

Supplementary figures and tables. [DOCX File, 309 KB-Multimedia Appendix 1]

\section{Multimedia Appendix 2}

Self-sampling instruction video.

[MP4 File (MP4 Video), 60416 KB-Multimedia Appendix 2]

\section{References}

1. Laboratory testing for coronavirus disease 2019 (COVID-19) in suspected human cases: interim guidance, 2 March 2020. World Health Organization. 2020. URL: https://apps.who.int/iris/handle/10665/331329 [accessed 2021-12-15] 
2. Fitzpatrick MC, Pandey A, Wells CR, Sah P, Galvani AP. Buyer beware: inflated claims of sensitivity for rapid COVID-19 tests. Lancet 2021 Jan 02;397(10268):24-25 [FREE Full text] [doi: 10.1016/S0140-6736(20)32635-0] [Medline: 33333002]

3. Würstle S, Spinner CD, Voit F, Hoffmann D, Hering S, Weidlich S, et al. Self-sampling versus health care professional-guided swab collection for SARS-CoV-2 testing. Infection 2021 Oct;49(5):927-934 [FREE Full text] [doi: 10.1007/s15010-021-01614-9] [Medline: 33970430]

4. Wehrhahn MC, Robson J, Brown S, Bursle E, Byrne S, New D, et al. Self-collection: An appropriate alternative during the SARS-CoV-2 pandemic. J Clin Virol 2020 Jul;128:104417 [FREE Full text] [doi: 10.1016/j.jcv.2020.104417] [Medline: 32403007]

5. Tu Y, Jennings R, Hart B, Cangelosi GA, Wood RC, Wehber K, et al. Swabs collected by patients or health care workers for SARS-CoV-2 testing. N Engl J Med 2020 Jul 30;383(5):494-496 [FREE Full text] [doi: 10.1056/NEJMc2016321] [Medline: 32492294]

6. Shakir SM, Barker AP, Hillyard DR, Gilmore N, Barrett JW, Orlandi RR, et al. Combined self-collected anterior nasal and oropharyngeal specimens versus provider-collected nasopharyngeal swabs for the detection of SARS-CoV-2. J Clin Microbiol 2020 Dec 17;59(1):e02291-e02220 [FREE Full text] [doi: 10.1128/JCM.02291-20] [Medline: 33087437]

7. McCulloch DJ, Kim AE, Wilcox NC, Logue JK, Greninger AL, Englund JA, et al. Comparison of Unsupervised Home Self-collected Midnasal Swabs With Clinician-Collected Nasopharyngeal Swabs for Detection of SARS-CoV-2 Infection. JAMA Netw Open 2020 Jul 01;3(7):e2016382 [FREE Full text] [doi: 10.1001/jamanetworkopen.2020.16382] [Medline: 32697321]

8. Goyal S, Prasert K, Praphasiri P, Chittaganpitch M, Waicharoen S, Ditsungnoen D, et al. The acceptability and validity of self-collected nasal swabs for detection of influenza virus infection among older adults in Thailand. Influenza Other Respir Viruses 2017 Sep;11(5):412-417 [FREE Full text] [doi: 10.1111/irv.12471] [Medline: 28741903]

9. Hart SG. Nasa-Task Load Index (NASA-TLX); 20 Years Later. Proceedings of the Human Factors and Ergonomics Society Annual Meeting 2016 Nov 05;50(9):904-908 [FREE Full text] [doi: 10.1177/154193120605000909]

10. Lu X, Wang L, Sakthivel SK, Whitaker B, Murray J, Kamili S, et al. US CDC real-time reverse transcription PCR panel for detection of severe acute respiratory syndrome coronavirus 2. Emerg Infect Dis 2020 Aug;26(8):1654-1665 [FREE Full

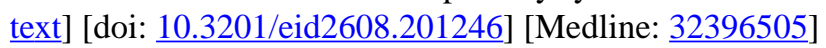

11. Wei WE, Li Z, Chiew CJ, Yong SE, Toh MP, Lee VJ. Presymptomatic transmission of SARS-CoV-2 - Singapore, January 23-March 16, 2020. MMWR Morb Mortal Wkly Rep 2020 Apr 10;69(14):411-415 [FREE Full text] [doi:

10.15585/mmwr.mm6914e1] [Medline: 32271722]

12. Casey M, Griffin J, McAloon C, Byrne A, Madden J, Mc ED, et al. Pre-symptomatic transmission of SARS-CoV-2 infection: a secondary analysis using published data. medRxiv. Preprint posted online on June 11, 2020 [FREE Full text] [doi: $10.1101 / 2020.05 .08 .20094870]$

13. Buitrago-Garcia D, Egli-Gany D, Counotte MJ, Hossmann S, Imeri H, Ipekci AM, et al. Occurrence and transmission potential of asymptomatic and presymptomatic SARS-CoV-2 infections: A living systematic review and meta-analysis. PLoS Med 2020 Sep;17(9):e1003346 [FREE Full text] [doi: 10.1371/journal.pmed.1003346] [Medline: 32960881]

14. Singanayagam A, Patel M, Charlett A, Lopez Bernal J, Saliba V, Ellis J, et al. Duration of infectiousness and correlation with RT-PCR cycle threshold values in cases of COVID-19, England, January to May 2020. Euro Surveill 2020 Aug;25(32):2001483 [FREE Full text] [doi: 10.2807/1560-7917.ES.2020.25.32.2001483] [Medline: 32794447]

15. Tan SY, Tey HL, Lim ETH, Toh ST, Chan YH, Tan PT, et al. The accuracy of healthcare worker versus self collected (2-in-1) oropharyngeal and bilateral mid-turbinate (OPMT) swabs and saliva samples for SARS-CoV-2. PLoS One 2020;15(12):e0244417 [FREE Full text] [doi: 10.1371/journal.pone.0244417] [Medline: 33326503 ]

16. Würstle S, Erber J, Hanselmann M, Hoffmann D, Werfel S, Hering S, et al. Simulation Tool for the Evaluation of Covid-19 Test Strategies. R. "Shiny" app. URL: https://mh-php-tum.shinyapps.io/Covid_19_Test_Strategy_Simulation/ [accessed 2021-12-15]

\section{Abbreviations}

CTM: Clinical Virus Transport Medium

IT: information technology

NAAT: nucleic acid amplification test

NASA: National Aeronautics and Space Administration

PCR: polymerase chain reaction

QR: quick response

UN 3373: United Nations' recommendation of dangerous goods 
Edited by G Eysenbach; submitted 02.08.21; peer-reviewed by A Zink, Y Man; comments to author 11.10.21; revised version received 18.10.21; accepted 21.11.21; published 04.01.22

Please cite as:

Würstle S, Erber J, Hanselmann M, Hoffmann D, Werfel S, Hering S, Weidlich S, Schneider J, Franke R, Maier M, Henkel AG, Schmid RM, Protzer $U$, Laxy $M$, Spinner $C D$

A Telemedicine-Guided Self-Collection Approach for PCR-Based SARS-CoV-2 Testing: Comparative Study

JMIR Form Res 2022;6(1):e32564

URL: https://formative.jmir.org/2022/1/e32564

doi: $10.2196 / 32564$

PMID: 34803022

(CSilvia Würstle, Johanna Erber, Michael Hanselmann, Dieter Hoffmann, Stanislas Werfel, Svenja Hering, Simon Weidlich, Jochen Schneider, Ralf Franke, Michael Maier, Andreas G Henkel, Roland M Schmid, Ulrike Protzer, Michael Laxy, Christoph D Spinner. Originally published in JMIR Formative Research (https://formative.jmir.org), 04.01.2022. This is an open-access article distributed under the terms of the Creative Commons Attribution License (https://creativecommons.org/licenses/by/4.0/), which permits unrestricted use, distribution, and reproduction in any medium, provided the original work, first published in JMIR Formative Research, is properly cited. The complete bibliographic information, a link to the original publication on https://formative.jmir.org, as well as this copyright and license information must be included. 